\title{
Membrane fouling in a submerged membrane bioreactor treating high strength municipal wastewater
}

\begin{abstract}
Studying the influence of the operating parameters on membrane fouling is important in fouling control. This paper presents the interaction effects of the sludge retention time (SRT), organic loading rate (OLR) and feed temperature (Tf) on membrane fouling. A submerged membrane bioreactor (SMBR) was operated under a constant flux $(11.1 \mathrm{l} / \mathrm{m} 2 \mathrm{~h}$ ), with different SRT, Tf and OLR. A synthetic wastewater representative of high strength municipal wastewater was fed to the bioreactor. Three different levels (low, medium and high) of SRT, Tf and OLR were studied. These were 25, 30 and 35 days for SRT, 20, 30 and $40^{\circ} \mathrm{C}$ for Tf and OLR of $1.73,4.036 .82 \mathrm{~kg} \mathrm{COD} / \mathrm{m} 3 . \mathrm{d}$. The sustainable time (tsus) was defined as the time at which the rate of suction pressure started to increase rapidly. tsus was found to increase with low SRT and high Tf. A higher OLR resulted in higher mixed liquor suspended solids (MLSS), however it did not cause a faster membrane fouling. Applying higher aeration rate enabled a longer sustainable time to be obtained. Sustainable time tsus was found to be well correlated with MLVSS/MLSS with an $\mathrm{r} 2$ of 0.995 . The range of MLVSS/MLSS tested varied from 74.3 to $82.3 \%$ at which tsus decreased 2.9-fold (from 175 to $60.5 \mathrm{~h}$ ).
\end{abstract}

Keyword: Membrane bioreactor (MBR); Membrane fouling; Sustainable time (tsus); Sludge retention time (SRT); Feed temperature (Tf); Organic loading rate (OLR) 